\title{
25 Research Square \\ Physiological uterine uptake of 68Ga-FAPI-04 may limit its application in uterine pathology
}

\section{Xiao Zhang}

Wuhan Union Hospital

Wenyu Song

Wuhan Union Hospital

Chunxia Qin

Wuhan Union Hospital

Yangmeihui Song

Wuhan Union Hospital

Fang Liu

Wuhan Union Hospital

Fan $\mathrm{Hu}$

Wuhan Union Hospital

Xiaoli Lan ( $\nabla$ xiaoli_lan@hust.edu.cn )

Union Hospital, Tongji Medical College, Huazhong University of Science \&amp; Technology https://orcid.org/0000-0002-7263-7399

\section{Research Article}

Keywords: 68Ga-FAPI-04, uterus, physiological uptake, PET/MRI, 18F-FDG

Posted Date: February 24th, 2021

DOl: https://doi.org/10.21203/rs.3.rs-249929/v1

License: (c) (1) This work is licensed under a Creative Commons Attribution 4.0 International License.

Read Full License 


\section{Abstract}

\section{Purpose}

${ }^{68} \mathrm{Ga}$-labeled fibroblast activation protein inhibitor $\left({ }^{68} \mathrm{Ga}-\mathrm{FAPI}\right)$ has been useful in the imaging of desmoplastic reaction in different tumors, as well as fibrosis in inflammatory diseases. As we have found that most female patients showed avid uterine uptake of ${ }^{68} \mathrm{Ga}$-FAPI, we sought to further investigate the physiological uptake of ${ }^{68} \mathrm{Ga}$-FAPI by the uterus and analyze its characteristics in women of childbearing age, in perimenopause, and postmenopause.

\section{Methods}

We retrospectively reviewed the image data of female patients who had undergone whole-body ${ }^{68} \mathrm{Ga}-$ FAPI-04 PET/MRI at our institute between May 22 and December 16, 2020. The clinical information of the patients, including age, stage of menstrual cycle if present, and gynecologic history, was collected. Uterine volume was calculated from MR images. The characteristics of ${ }^{68} \mathrm{Ga}-\mathrm{FAPI}-04$ uterine uptake were recorded. If the patients also had undergone ${ }^{18} \mathrm{~F}$-FDG PET/CT imaging, those image features were also recorded. The relationship of age, uterine size, surgical history, presence of leiomyomas, and ${ }^{18} \mathrm{~F}-\mathrm{FDG}$ uptake to ${ }^{68} \mathrm{Ga}-\mathrm{FAPI}-04$ uptake were further analyzed.

Results

Forty-three female patients were included in this study. The number of cases with uterine malignancy and non-malignancy were two and 41, respectively. Twenty-seven patients in the benign disease group underwent simultaneous ${ }^{18}$ F-FDG PET/CT scans. Of 41 patients with benign uterine pathology, 13 patients were of reproductive age, six were perimenopausal, and 22 were postmenopausal. A total of 25 patients had undergone invasive operations, and six patients had uterine fibroids under examination. Lower ${ }^{68} \mathrm{Ga}$-FAPI-04 uptake $(6.58 \pm 3.39, n=22)$ was noted in postmenopausal women than in reproductive and perimenopausal females $(13.32 \pm 3.2$ and $12.98 \pm 3.01$, respectively, $P<0.05)$. The invasive operation or accompany with uterine fibroids may increase ${ }^{68} \mathrm{Ga}-\mathrm{FAPI}-04$ uptake. ${ }^{68} \mathrm{Ga}-\mathrm{FAPI}-04$ uptake was also associated with uterine volume $(P<0.01)$. There was no correlation between ${ }^{18} \mathrm{~F}$-FDG and ${ }^{68} \mathrm{Ga}$-FAPI-04 accumulation in the uterus. Two patients with malignant lesions involving the uterus showed completely different imaging features on ${ }^{68} \mathrm{Ga}-\mathrm{FAPI}-04$.

\section{Conclusion}

Physiological uterine uptake of ${ }^{68} \mathrm{Ga}$-FAPI-04 limits its diagnostic value in gynecologic diseases. Age, uterine fibroids and uterine volume may influence the uptake of ${ }^{68} \mathrm{Ga}-\mathrm{FAPI}-04$ in uterus. More patients with various uterine diseases could be involved to provide more differential diagnostic information. 


\section{Introduction}

Fibroblast activation protein (FAP) is regarded as an excellent target for diagnosis and therapy in oncology $[1,2] .{ }^{68} \mathrm{Ga}$-labeled FAPI-04 (a specific inhibitor of FAP) is used as a PET tracer for tumor diagnosis by visualizing desmoplastic reaction $[3,4]$. It also has the potential for non-malignant diseases associated with tissue remodeling, such as myocardial infarction, sarcoidosis, chronic inflammation, fibrosis of lung and liver, rheumatoid arthritis, and possibly also atherosclerosis [5-10]. The clinical applications of ${ }^{68} \mathrm{Ga}-\mathrm{FAPI}-04$ PET in different diseases are being defined.

Some studies have reported the physiological changes in the female reproductive system using ${ }^{18} \mathrm{~F}-\mathrm{FDG}$ PET [11-13]. In our clinical studies to evaluate the diagnostic value of ${ }^{68} \mathrm{Ga}-\mathrm{FAPI}-04$, it occurred to us that elevated ${ }^{68} \mathrm{Ga}$-FAPI-04 uptake in the uterus were noted in most female patients. We reviewed the literature and case reports on clinical studies of ${ }^{68} \mathrm{Ga}-\mathrm{FAPI}-04$ and found that uterine uptake of the tracer is very common [14-16]. Uptake patterns varied from patient to patient. There are no further discussions of the physiological uptake of ${ }^{68} \mathrm{Ga}-\mathrm{FAPI}-04$ by the uterus. Primary and metastatic malignant uterine tumors are common [17]. The uptake of ${ }^{68} \mathrm{Ga}-\mathrm{FAPI}-04$ by the uterus may affect the evaluation of uterine lesions, and the factors related to uterine uptake are still lacking.

On the basis of the evaluation of all female patients in our institute who underwent ${ }^{68} \mathrm{Ga}-\mathrm{FAPI}-04$ $\mathrm{PET} / \mathrm{MRI}$, the aim of this study was to analyze the physiological uptake and characteristics of ${ }^{68} \mathrm{Ga}-\mathrm{FAPI}$ in the uterus, and to further evaluate its value in the diagnosis of uterine diseases.

\section{Material And Methods}

\section{Patients}

${ }^{68} \mathrm{Ga}$-FAPI-04 application in tumors and hepatic fibrosis were performed at our institute, which have been approved by the Institutional Review Board of Union Hospital, Tongji Medical College, Huazhong University of Science and Technology (NO. 20200290 and 20200507). The need for written informed consent was waived. The clinical trial registration numbers are NCT04605939 and NCT04554719. We retrospectively analyzed the imaging data of all 132 patients who underwent ${ }^{68} \mathrm{Ga}-\mathrm{FAPI}-04 \mathrm{PET} / \mathrm{MRI}$ from May 22 to December 16, 2020. The inclusion criteria for this study were (1) age $\geq 18$ years, (2) female, and (3) complete clinical and imaging data. We excluded patients who had a prior hysterectomy. All gynecologic history and menstrual phase data were collected by clinical data or follow-up investigation. The follow-up time was 2-8 months.

\section{${ }^{68} \mathrm{Ga}-\mathrm{FAPI}-04$ and ${ }^{18} \mathrm{~F}-\mathrm{FDG}$ imaging protocol}


${ }^{68} \mathrm{Ga}$-FAPI-04 labeling was performed according to a previously described protocol [18]. ${ }^{18} \mathrm{~F}-\mathrm{FDG}$ was synthesized with ${ }^{18} \mathrm{~F}$ produced by a cyclotron (MINItrace ${ }^{\circledR}, \mathrm{GE}$ Healthcare, Milwaukee, WI, USA). The radiochemical purity was over $95 \%$ for both ${ }^{68} \mathrm{Ga}-\mathrm{FAPI}-04$ and ${ }^{18} \mathrm{~F}-\mathrm{FDG}$.

A total of $1.8-2.2 \mathrm{MBq} / \mathrm{kg}{ }^{68} \mathrm{Ga}-\mathrm{FAPI}-04$ was administered intravenously. After the injection for $60 \mathrm{~min}$, the patients underwent a hybrid time-of-flight PET/MRI examination (SIGNA ${ }^{\mathrm{TM}} \mathrm{PET} / \mathrm{MRI}$, GE Healthcare, Waukesha, WI, USA). PET acquisition was performed in 3D mode with 15 min per bed position (DFOV = $30 \mathrm{~cm}$ ). Simultaneous with the PET acquisition, MR imaging protocols included T1-weighted imaging (T1WI), T2-weighted imaging (T2WI), T1-fat suppression (T1WI/FS), T2-fat suppression (T2WI/FS), and diffusion-weighted imaging (DWI).

${ }^{18} \mathrm{~F}-\mathrm{FDG}$ PET/CT was performed approximately $60 \mathrm{~min}$ after ${ }^{18} \mathrm{~F}-\mathrm{FDG}(3.7-5.5 \mathrm{MBq} / \mathrm{kg})$ administration (Discovery VCT ${ }^{\circledR}$, GE Healthcare).

\section{Image analysis}

All PET/MRI and PET/CT data were processed using Advanced Workstation (AW4.6, GE Healthcare) in DICOM format. ${ }^{68} \mathrm{Ga}$-FAPI-04 PET/MRI and ${ }^{18} \mathrm{~F}$-FDG PET/CT images were interpreted by three experienced nuclear medicine physicians. ${ }^{68} \mathrm{Ga}$-FAPI-04 uptake in the uterine fundus, body, and cervix were measured and expressed as maximum standardized uptake value (SUVmax) and mean standardized uptake value (SUVmean). Uterine volume was determined by longitudinal diameter $\times$ anteroposterior diameter $\times$ transverse diameter $\times 0.45$ on the MR images.

\section{Statistical analysis}

SPSS 19.0 (IBM Inc., Armonk, NY, USA) was used for data processing. Continuous variables are expressed as mean \pm SD. Analysis of variance (ANOVA) and Student's $t$-test was used for quantitative data with normal distribution and equal variances. The correlations of continuous variables (uterine size, age, ${ }^{18} \mathrm{~F}$ FDG and ${ }^{68} \mathrm{Ga}$-FAPI-04 uptake) were also analyzed with curve fit by linear, quadratic and logistic regression. $P<0.05$ was considered statistically significant.

\section{Results}

\section{Demographic and clinical characteristics of the patients}

From May 22 to December 16, a total of 52 female patients underwent whole-body ${ }^{68} \mathrm{Ga}$-FAPI-04 $\mathrm{PET} / \mathrm{MRI}$ at our institution. Nine patients with hysterectomy were excluded. There were two patients with uterine malignancy and 41 patients without uterine malignancy in this study. Twenty-nine patients had undergone both ${ }^{18} \mathrm{~F}$-FDG PET/CT and ${ }^{68} \mathrm{Ga}$-FAPI-04 PET/MRI scans within 1 week of each other (including 2 malignant patients). 
The flowchart and clinical information of the included patients are shown in Fig. 1 and Table 1, respectively. Patients without uterine malignancy were divided into three groups: 13 (mean age, $34.92 \pm$ 4.55 years) of reproductive age, six (mean age, $49.17 \pm 1.17$ years) in the perimenopausal stage, and 22 (mean age, $59.36 \pm 6.51$ years) who were postmenopausal. For females of reproductive age, 12 patients were in the luteal phase and one in the ovulatory phase at the time of examination. One of the perimenopausal patients was experiencing menses. From the participants without uterine malignancy (41), 25 (60.9\%) had a history of uterine invasive operation due to benign causes, including cesarean section $(14,34.1 \%)$, pregnancy termination $(7,17.1 \%)$, intrauterine device implantation $(4,9.8 \%)$, and myomectomy $(1,2.4 \%)$.

\section{Patterns of uterine uptake of ${ }^{68} \mathrm{Ga}-\mathrm{FAPI}-04$}

The ${ }^{68} \mathrm{Ga}-\mathrm{FAPI}-04$ and ${ }^{18} \mathrm{~F}-\mathrm{FDG}$ SUVmax of uterus and uterine volume among different groups are summarized in Table 2. Lower ${ }^{68} \mathrm{Ga}$-FAPI-04 uptake was noted in postmenopausal women compared with reproductive age and perimenopausal females. Higher accumulation of FAPI in the surgical group was found than that in patients with no surgical history $(P=0.047)$. Of the patients with leiomyomas, two were perimenopausal and four were postmenopausal. In the perimenopausal and postmenopausal

periods, the patients with leiomyomas showed higher ${ }^{68} \mathrm{Ga}-\mathrm{FAPI}-04$ uptake. The uterine fundus, body, and cervix had different uptake values (SUVmax 8.27 $\pm 3.71,9.44 \pm 4.59$ and $6.32 \pm 4.26$, respectively, $P<$ 0.001 , measured on coronal PET images).

\section{Imaging features of reproductive age, perimenopausal, and postmenopausal patients}

In Fig. 2, three patients in reproductive, perimenopausal, and menopausal periods showed varying degrees of FAPI uptake in the uterus. MRI and CT images showed the expected uterine volume decrease with increasing age. ${ }^{68} \mathrm{Ga}-\mathrm{FAPI}-04$ uptake was higher in patients of reproductive and perimenopausal stages than in postmenopausal patients (Fig. 2a-I). The images showed that the smallest uterine size and oldest age correlated with the lowest FAPI uterine uptake values. On ${ }^{18} \mathrm{~F}-\mathrm{FDG}$ PET/CT scans, the uterus had low FDG uptake, with no significant differences among individuals (Fig. $\mathbf{2 m - x}$ ). In addition, mild uptake was noted in the ovaries in this set of patients (yellow arrows).

\section{The uterus with fibroids or invasive operation}

In Fig. 3a-d, when the uterus was involved by fibroids (red arrows), the uptake of ${ }^{68} \mathrm{Ga-FAPI} 04$ increased, especially in the fibroid margin and myometrium. As shown in Fig. $\mathbf{3 e}-\mathbf{h}$, an intrauterine device (IUD, yellow arrows) was associated with intense uptake, implying that patients who had undergone invasive procedures may increase ${ }^{68} \mathrm{Ga}-\mathrm{FAPI}-04$ uptake around the site.

\section{The uterus with primary or secondary malignancy}

In our clinical study, there were two patients with malignancy. The patient (Fig. 4) with metastases to the uterus showed a lesion with relatively low ${ }^{68} \mathrm{Ga}-\mathrm{FAPI}-04$ uptake, which was opposite to the avid 
accumulation of ${ }^{18} \mathrm{~F}-\mathrm{FDG}$. The case (Fig. 5) of endometrial adenocarcinoma had intense uptake of ${ }^{68} \mathrm{Ga}-$ FAPI-04.

\section{Various regression analyses}

Linear, quadratic, and logistic regression equations were used to assess for correlations among age, uterine size, ${ }^{18} \mathrm{~F}$-FDG uptake, and ${ }^{68} \mathrm{Ga}$-FAPI-04 uptake (Fig. 6). For uterine volume and ${ }^{68} \mathrm{Ga}-\mathrm{FAPI}-04$ uptake, the linear regression equation parameters and percentage of variance accounted for $R^{2}$ were $y=$ $0.065 x+4.192$ and adjusted $R^{2}=0.444(P<0.01)$, respectively (Fig. 6a). For age and ${ }^{68} \mathrm{Ga}$-FAPI-04 uptake, the linear regression equation was $y=21.67-0.24 x$ and adjusted $R^{2}=0.389(P<0.01$; Fig. 6b). It showed that ${ }^{68} \mathrm{Ga}$-FAPI-04 uptake was positively associated with uterine volume and negatively associated with age. For uterine size and ${ }^{18} \mathrm{~F}$-FDG uptake, ${ }^{18} \mathrm{~F}-\mathrm{FDG}$ and ${ }^{68} \mathrm{Ga}$-FAPI-04 uptake, the points were randomly distributed throughout the plot. There were no correlations between uterine size and ${ }^{18} \mathrm{~F}-$ FDG accumulation, or between ${ }^{18} \mathrm{~F}-\mathrm{FDG}$ and ${ }^{68} \mathrm{Ga}$-FAPI-04 accumulation $(P>0.05$; Fig. $6 \mathrm{c}$ and d).

\section{Discussion}

As a new and promising tumor imaging agent, ${ }^{68} \mathrm{Ga}-\mathrm{FAPI}-04$ has been gradually applied in diagnosing and staging of tumors [19]. However, avid ${ }^{68} \mathrm{Ga}$-FAPI-04 uptake in the uterus of adult women may limit the detection of uterine lesions. Therefore, this study aimed to investigate the accumulation of ${ }^{68} \mathrm{Ga}-\mathrm{FAPI}-04$ in the uterus. We found that FAPI uptake is related to age and uterine volume. Prior surgery and the presence of fibroids may increase ${ }^{68} \mathrm{Ga}$-FAPI-04 accumulation. FAPI uptake is lower in the normal postmenopausal uterus. Different uptake features were found in two uterine malignancies, one primary and one secondary. To the best of our knowledge, this is the first detail analysis of the uterine uptake on ${ }^{68} \mathrm{Ga}-\mathrm{FAPI}-04$ PET/MRI.

In our study, most female patients showed varying degrees of FAPI uptake in the uterus. The adult uterus is a thick walled, hollow, muscular organ [20]. FAP provides required support for muscle fiber maturation and specification [21-23]. Higher accumulations were noted in the uteri of patients of reproductive age and in the perimenopausal period compared with postmenopausal patients. The high uterine FAP activity may result from the tissue remodeling and angiogenesis during the menstrual cycle [23-25]. Low uptake was noted in the ovaries, possibly due to the hormone fluctuation mainly acting on follicles rather than mesenchyme [26, 27], which also supports the idea that ${ }^{68}$ Ga-FAPI-04 PET/MRI may be useful in the diagnosis of ovarian diseases.

Larger uteri had higher ${ }^{68} \mathrm{Ga}-\mathrm{FAPI}-04$ uptake. The size of the uterus corresponds with the thickness of the myometrium $[24,28]$. Women who undergone uterine surgery had higher uterine uptake of ${ }^{68} \mathrm{Ga}-\mathrm{FAPI}-04$. This could be due to the role played by FAP in wound healing [29], which may increase the binding of FAPI. ${ }^{68} \mathrm{Ga}-\mathrm{FAPI}-04$ uptake was also increased in postmenopausal and perimenopausal patients with fibroids, especially in the margin and surroundings of the fibroid. The growth of fibroids is an active 
process of fibrogenesis and relies on the expansion of surrounding mature myometrial and fibroid cells $[30,31]$.

The two malignant cases showed completely different features. In the first case, one of metastatic disease, the activity in the lesion was decreased compared with normal uterine tissue. For the other patient with primary endometrial cancer, intensely increased and diffuse uptake involved the entire uterus, which may be confused with physiological uptake. Although the ${ }^{68} \mathrm{Ga}-\mathrm{FAPI}-04 \mathrm{PET} / \mathrm{MRI}$ displayed the tumor, it does not appear suitable for distinguishing uterine tumors. It appears that high SUVs do not improve the accuracy in assessing whether a tumor has extended or metastasized to the uterus. Combined with ${ }^{18} \mathrm{~F}-\mathrm{FDG}$ and MR images, it may improve the diagnostic efficacy of the malignancy in the uterus.

There are several limitations in this study. First, the impact of the menstrual cycle on ${ }^{68} \mathrm{Ga}$-FAPI-04 uptake is worth exploring, as most patients of reproductive age in this group were in the luteal phase. Therefore, more patients in different menstrual phases should be investigated. Second, sex hormone should be examined to provides evidence to support the relationship between sex hormone and FAPI uptake in uterus. Last, the number of patients with uterine malignancy was small, which limited the statistical analysis to distinguish between malignant and benign uptake.

In this preliminary study, our findings suggest that ${ }^{68} \mathrm{Ga}$-FAPI-04 PET may not be a suitable tool for the diagnosis of uterine disease because of its high physiological uptake by the uterus. Increased uterine uptake may be associated with age, uterine volume, surgical history, and the presence of fibroids. Because differing features of ${ }^{68} \mathrm{Ga}$-FAPI-04 were found in the malignant lesions, a prospective study with a larger sample size is necessary for further evaluating the clinical value of ${ }^{68} \mathrm{Ga}$-FAPI-04 PET/MRI in the uterine diseases.

\section{Declarations}

\section{Funding}

This work was supported by National Natural Science Foundation of China (No. 82030052, and 81901783).

\section{Conflict of interest}

The authors declare that they have no conflict of interest.

\section{Ethical approval}

All procedures involving human participants were carried out in accordance with the ethical standards of the institutional and/or national research committee and with the 1964 Helsinki Declaration and its later amendments or comparable ethical standards. 


\section{Consent to participate and publication}

The requirement for informed consent was waived.

\section{Availability of data and code}

Not applicable.

\section{Authors' contributions}

1. L. conceived and designed the study devised, supervised the project. X. Z. and W. S. wrote the manuscript. C. Q. and F. L. analyzed the image data. Y. M. and F. H. contributed to the follow-up of the patients.

\section{References}

1. Langbein T, Weber WA, Eiber M. Future of Theranostics: An Outlook on Precision Oncology in Nuclear Medicine. J Nucl Med. 2019;60(Suppl 2):13S-9S. doi:10.2967/jnumed.118.220566.

2. Hamson EJ, Keane FM, Tholen S, Schilling O, Gorrell MD. Understanding fibroblast activation protein (FAP): substrates, activities, expression and targeting for cancer therapy. Proteomics Clin Appl. 2014;8(5-6):454-63. doi:10.1002/prca.201300095.

3. Toms J, Kogler J, Maschauer S, Daniel C, Schmidkonz C, Kuwert T et al. Targeting Fibroblast Activation Protein: Radiosynthesis and Preclinical Evaluation of an (18)F-labeled FAP Inhibitor. J Nucl Med. 2020. doi:10.2967/jnumed.120.242958.

4. Lindner T, Loktev A, Altmann A, Giesel F, Kratochwil C, Debus J et al. Development of Quinoline-Based Theranostic Ligands for the Targeting of Fibroblast Activation Protein. J Nucl Med. 2018;59(9):141522. doi:10.2967/jnumed.118.210443.

5. Pan Q, Luo Y, Zhang W. Recurrent Immunoglobulin G4-Related Disease Shown on 18F-FDG and 68Ga-FAPI PET/CT. Clin Nucl Med. 2020;45(4):312-3. doi:10.1097/RLU.0000000000002919.

6. Zhang X, Song W, Qin C, Liu F, Lan X. Non-malignant findings of focal (68)Ga-FAPI-04 uptake in pancreas. Eur J Nucl Med Mol Imaging. 2021. doi:10.1007/s00259-021-05194-6.

7. Schmidkonz C, Rauber S, Atzinger A, Agarwal R, Gotz TI, Soare A et al. Disentangling inflammatory from fibrotic disease activity by fibroblast activation protein imaging. Ann Rheum Dis. 2020;79(11):1485-91. doi:10.1136/annrheumdis-2020-217408.

8. Luo Y, Pan Q, Zhang W. IgG4-related disease revealed by (68)Ga-FAPI and (18)F-FDG PET/CT. Eur J Nucl Med Mol Imaging. 2019;46(12):2625-6. doi:10.1007/s00259-019-04478-2.

9. Zheng J, Yao S. [(68)Ga]Ga-DOTA-FAPI-04 and [(18)F] FDG PET/CT for the diagnosis of primary and metastatic lesions in patients with hepatic cancer. Eur J Nucl Med Mol Imaging. 2020;47(9):2078-9. doi:10.1007/s00259-020-04847-2. 
10. Heckmann MB, Reinhardt F, Finke D, Katus HA, Haberkorn U, Leuschner F et al. Relationship Between Cardiac Fibroblast Activation Protein Activity by Positron Emission Tomography and Cardiovascular Disease. Circ Cardiovasc Imaging. 2020;13(9):e010628. doi:10.1161/CIRCIMAGING.120.010628.

11. Nishizawa S, Inubushi M, Okada H. Physiological 18F-FDG uptake in the ovaries and uterus of healthy female volunteers. Eur J Nucl Med Mol Imaging. 2005;32(5):549-56. doi:10.1007/s00259004-1703-x.

12. Even-Sapir E. Imaging the Normal and Abnormal Anatomy of the Female Pelvis Using (18)F FDGPET/CT, Including Pitfalls and Artifacts. PET Clin. 2010;5(4):425-34. doi:10.1016/j.cpet.2010.07.003.

13. Lerman H, Metser U, Grisaru D, Fishman A, Lievshitz G, Even-Sapir E. Normal and abnormal F-18-FDG endometrial and ovarian uptake in pre- and postmenopausal patients: Assessment by PET/CT. J Nucl Med. 2004;45(2):266-71.

14. Qin C, Gai Y, Liu Q, Shao F, Lan X. Elevated 68Ga-FAPI Accumulation in a Recurrent Angiomyolipoma. Clin Nucl Med. 2020;45(12):1034-5. doi:10.1097/RLU.0000000000003345.

15. Sonni I, Lee-Felker S, Memarzadeh S, Quinn MM, Mona CE, Luckerath K et al. (68)Ga-FAPi-46 diffuse bilateral breast uptake in a patient with cervical cancer after hormonal stimulation. Eur J Nucl Med Mol Imaging. 2020. doi:10.1007/s00259-020-04947-z.

16. Shi $X$, Xing H, Yang X, Li F, Yao S, Zhang $H$ et al. Fibroblast imaging of hepatic carcinoma with (68)Ga-FAPI-04 PET/CT: a pilot study in patients with suspected hepatic nodules. Eur J Nucl Med Mol Imaging. 2020. doi:10.1007/s00259-020-04882-z.

17. Siegel RL, Miller KD, Jemal A. Cancer statistics, 2020. CA Cancer J Clin. 2020;70(1):7-30. doi:10.3322/caac. 21590 .

18. Chen H, Zhao L, Ruan D, Pang Y, Hao B, Dai Y et al. Usefulness of [(68)Ga]Ga-DOTA-FAPI-04 PET/CT in patients presenting with inconclusive [(18)F]FDG PET/CT findings. Eur J Nucl Med Mol Imaging. 2020. doi:10.1007/s00259-020-04940-6.

19. Pang Y, Zhao L, Luo Z, Hao B, Wu H, Lin Q et al. Comparison of 68Ga-FAPI and 18F-FDG Uptake in Gastric, Duodenal, and Colorectal Cancers. Radiology. 2021;298(2):393-402. doi:10.1148/radiol.2020203275.

20. La Rosa PS, Eswaran H, Preissl H, Nehorai A. Multiscale forward electromagnetic model of uterine contractions during pregnancy. BMC Med Phys. 2012;12:4-. doi:10.1186/1756-6649-12-4.

21. Luo N, Guan Q, Zheng L, Qu X, Dai H, Cheng Z. Estrogen-mediated activation of fibroblasts and its effects on the fibroid cell proliferation. Transl Res. 2014;163(3):232-41. doi:10.1016/j.trsl.2013.11.008.

22. Keane FM, Yao TW, Seelk S, Gall MG, Chowdhury S, Poplawski SE et al. Quantitation of fibroblast activation protein (FAP)-specific protease activity in mouse, baboon and human fluids and organs. FEBS Open Bio. 2013;4:43-54. doi:10.1016/j.fob.2013.12.001.

23. Santos AM, Jung J, Aziz N, Kissil JL, Pure E. Targeting fibroblast activation protein inhibits tumor stromagenesis and growth in mice. J Clin Invest. 2009;119(12):3613-25. doi:10.1172/JCI38988. 
24. Well D, Yang H, Houseni M, Iruvuri S, Alzeair S, Sansovini M et al. Age-related structural and metabolic changes in the pelvic reproductive end organs. Semin Nucl Med. 2007;37(3):173-84. doi:10.1053/j.semnuclmed.2007.01.004.

25. Orbak Z, Sagsoz N, Alp H, Tan H, Yildirim H, Kaya D. Pelvic ultrasound measurements in normal girls: Relation to puberty and sex hormone concentration. J Pediatr Endocrinol Metab. 1998;11(4):525-30.

26. Madekurozwa MC, Oke BO, Akinloye AK. The immunohistochemical localization of desmin and smooth muscle actin in the ovary of the African giant rat (Cricetomys gambianus) during the oestrous cycle. Anat Histol Embryol. 2010;39(1):81-6. doi:10.1111/j.1439-0264.2009.00983.x.

27. Channing CP, Schaerf FW, Anderson LD, Tsafriri A. Ovarian follicular and luteal physiology. Int Rev Physiol. 1980;22:117-201.

28. Hart R, Sloboda DM, Doherty DA, Norman RJ, Atkinson HC, Newnham JP et al. Prenatal determinants of uterine volume and ovarian reserve in adolescence. J Clin Endocrinol Metab. 2009;94(12):4931-7. doi:10.1210/jc.2009-1342.

29. Chen H, Pang Y, Wu J, Zhao L, Hao B, Wu J et al. Comparison of [(68)Ga]Ga-DOTA-FAPI-04 and [(18)F] FDG PET/CT for the diagnosis of primary and metastatic lesions in patients with various types of cancer. Eur J Nucl Med Mol Imaging. 2020;47(8):1820-32. doi:10.1007/s00259-020-04769-z.

30. Malik M, Britten J, Catherino WH. Development and Validation of Hormonal Impact of a Mouse Xenograft Model for Human Uterine Leiomyoma. Reprod Sci. 2020;27(6):1304-17. doi:10.1007/s43032-019-00123-3.

31. Moravek MB, Bulun SE. Endocrinology of uterine fibroids: steroid hormones, stem cells, and genetic contribution. Curr Opin Obstet Gynecol. 2015;27(4):276-83. doi:10.1097/GC0.0000000000000185.

\section{Tables}

Table 1 The gynecologic status of non-malignant patients 


\begin{tabular}{|l|l|}
\hline Patients & Total \\
\hline Age (years) & $50.12 \pm 12.28$ \\
\hline Phase & $(n$, years $)$ \\
\hline Reproductive period & $13,34.92 \pm 4.55$ \\
\hline Perimenopausal period & $6,49.17 \pm 1.17$ \\
\hline Postmenopausal period & $22,59.36 \pm 6.51$ \\
\hline Gynecology surgery (n) & 25 \\
\hline Cesarean operation & 14 \\
\hline Induced abortion & 7 \\
\hline Intrauterine device implantation & 4 \\
\hline Myomectomy & 1 \\
\hline No gynecology surgery (n) & 16 \\
\hline Leiomyoma (n) & 6 \\
\hline Contemporaneous ${ }^{18}$ F-FDG PET/CT (n) & 27 \\
\hline
\end{tabular}

Table 2 The SUVmax and uterine volume among different patient groups 


\begin{tabular}{|llll|}
\hline Patients & ${ }^{68}$ Ga-FAPI-04 SUVmax & ${ }^{18}$ F-FDG & Uterine volume $\left(\mathrm{cm}^{3}\right)$ \\
\hline Total & & & \\
\hline Average $(n)$ & $9.65 \pm 4.63(41)$ & $3.10 \pm 1.39(27)$ & $83.82 \pm 48.11(41)$ \\
\hline Phase & & & \\
\hline Reproductive period $(n=13)$ & $13.32 \pm 3.20$ & $2.80 \pm 1.24$ & $101.65 \pm 40.36$ \\
\hline Perimenopausal period $(n=6)$ & $12.98 \pm 3.01$ & $4.30 \pm 1.66$ & $118.70 \pm 57.17$ \\
\hline Postmenopausal period $(n=22)$ & $6.58 \pm 3.39$ & $2.84 \pm 1.22$ & $64.77 \pm 41.66$ \\
\hline F/P & $20.794 / 0.000$ & $2.603 / 0.095$ & $5.327 / 0.009$ \\
\hline With/without operation & & & \\
\hline Invasive operation $(n=25)$ & $10.80 \pm 4.60$ & $3.38 \pm 1.55$ & $95.05 \pm 51.55$ \\
\hline None invasive operation $(n=16)$ & $7.87 \pm 4.22$ & $2.53 \pm 0.79$ & $66.27 \pm 37.19$ \\
\hline T/P & $2.052 / 0.047$ & $1.531 / 0.138$ & $1.931 / 0.061$ \\
\hline With/without fibroid* & & & \\
\hline Fibroid $(n=6)$ & $11.12 \pm 4.38$ & $4.20 \pm 1.76$ & $120.40 \pm 68.82$ \\
\hline Without fibroid $(n=22)$ & $7.09 \pm 3.83$ & $2.97 \pm 1.31$ & $63.30 \pm 36.49$ \\
\hline T/P & $2.219 / 0.035$ & $1.538 / 0.144$ & $1.975 / 0.100^{\#}$ \\
\hline
\end{tabular}

*refers to perimenopausal and postmenopausal patients.

\# refers to Welch's $t$-test.

\section{Figures}




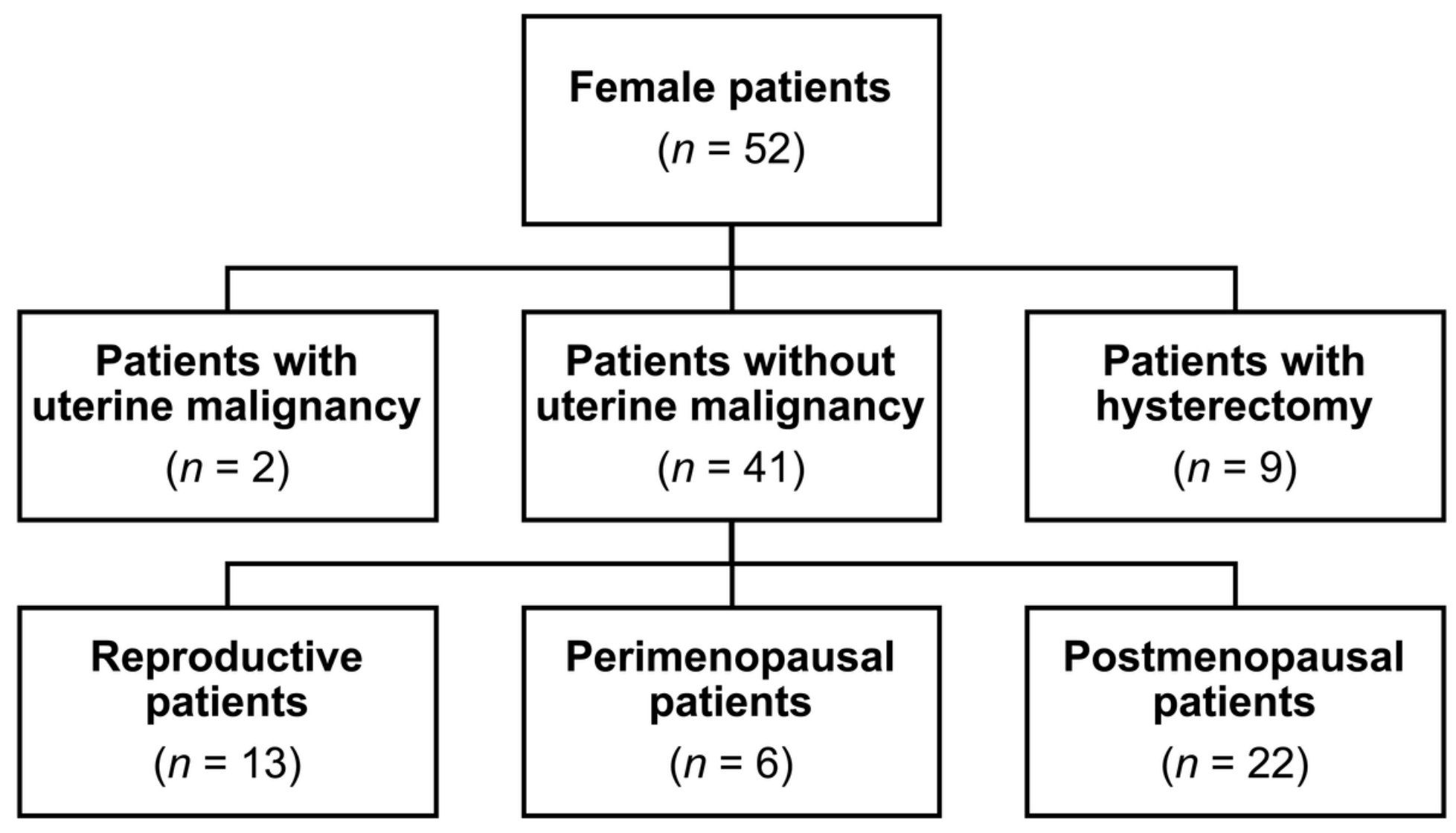

Figure 1

The flow diagram of enrolled patients
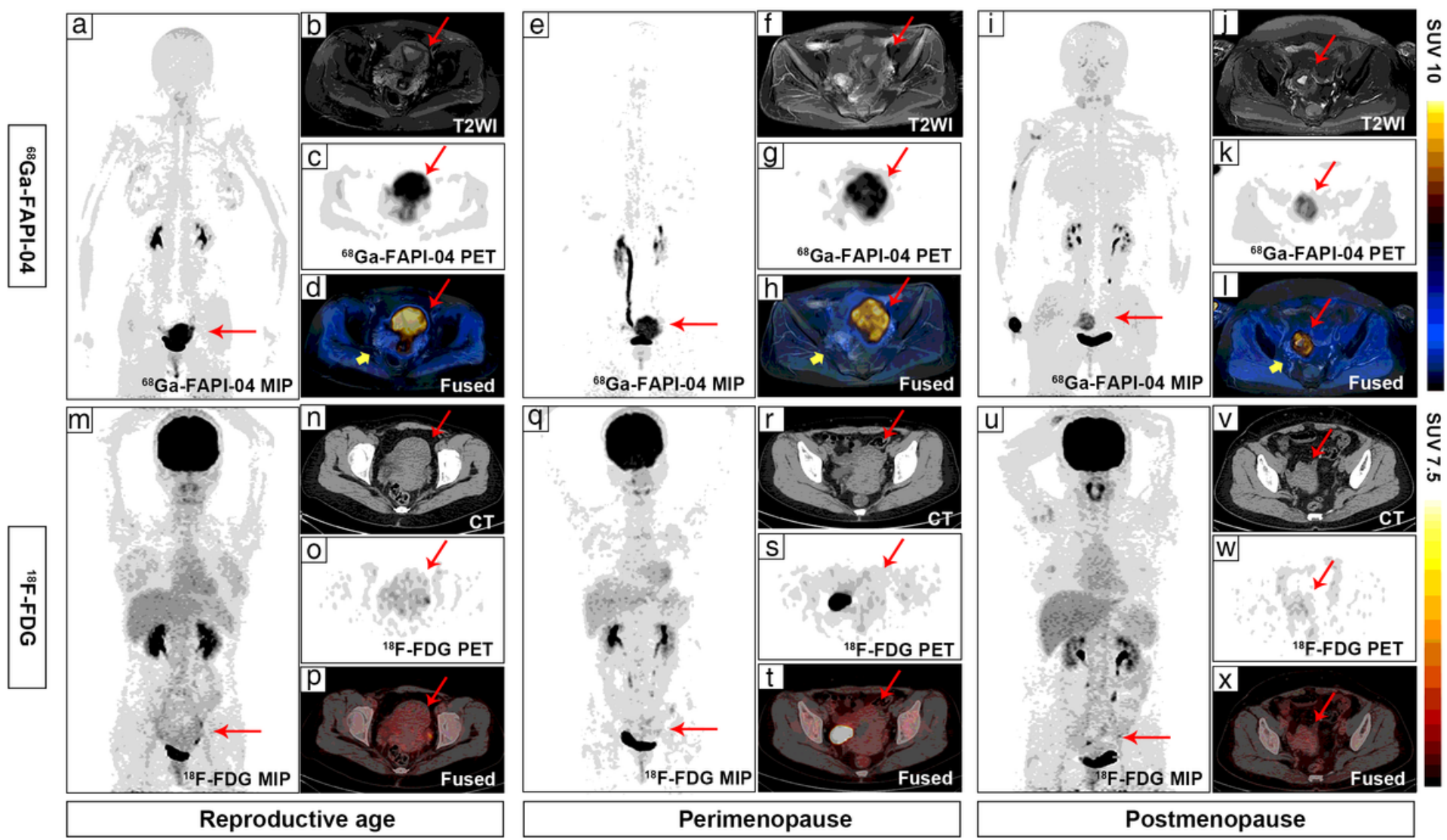


\section{Figure 2}

68Ga-FAPI-04 PET/MRI and 18F-FDG PET/CT images of women of reproductive age, in the perimenopausal period, and in the postmenopausal period. A 38-year-old woman of reproductive age had avid 68Ga-FAPI-04 uptake in the uterus (SUVmax 11.9) and the breasts (SUVmax 4.5) on a maximum intensity projection (MIP) image (a). MR scan (b) demonstrated a 112.44-cm3 uterine volume. The corresponding 18F-FDG images $(\mathrm{m}-\mathrm{p}$ ) of the uterus showed slight accumulation (SUVmax 3.3). A 48year-old patient showed avid 68Ga-FAPI-04 accumulation (SUVmax 16.3) in the uterus (e), while low uptake (SUVmax 3.5) of 18F-FDG (q). The uterine structure was clearly delineated by T2WI/FS image (f, volume $=136.18 \mathrm{~cm} 3) .68 \mathrm{Ga}-\mathrm{FAPI}-04 \mathrm{PET}$ images showed low uterine uptake (i, $\mathrm{k}$ and I, SUVmax 7.6) in a 42-year-old woman, and 18F-FDG PET/CT images $(u-x)$ with an SUVmax value of 6.1. Abnormal changes in the uterus were not found on T2WI/FS (j, volume $=63.40 \mathrm{~cm} 3)$ and CT $(w)$ images. The right upper arm focus (i) was due to extravasation of the injection. The red and yellow arrows refer to the uterus and ovaries, respectively.
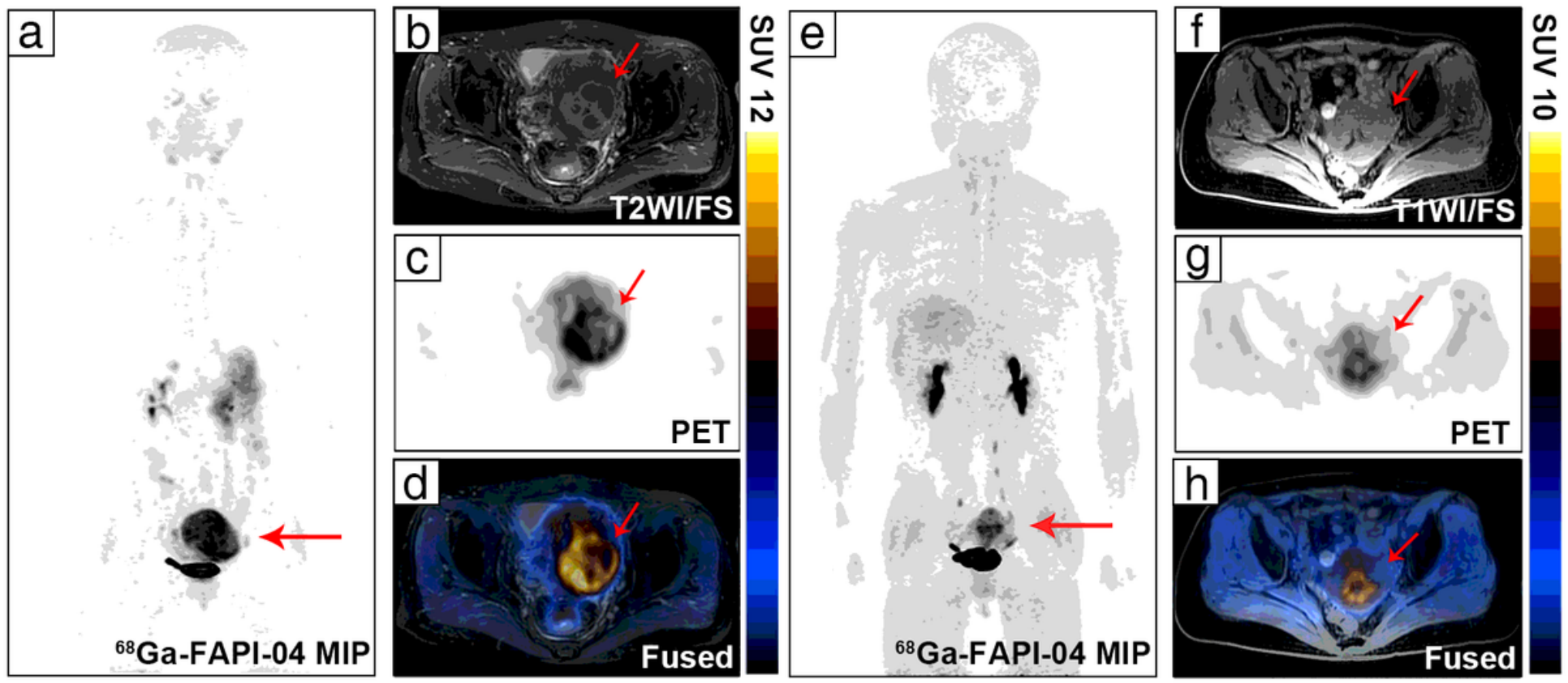

\section{Figure 3}

68Ga-FAPI-04 imaging of patients with uterine fibroids and an intrauterine device (IUD). The MIP image (a) of 68Ga-FAPI-04 showed intense uptake in the uterus (red arrow). T2WI/FS image demonstrated uterine leiomyoma of mixed signal intensity with internal septations ( $b$, red arrow). The uterine tissue squeezed by the fibroids was slightly displaced, and showed high uptake of FAPI (SUVmax 16.7, c and d, red arrows). Avid uterine accumulation was noted in the MIP image of a 53-year-old woman (e, yellow arrow). An IUD was visualized on T1WI as an area of hypointense signal (f). Elevated 68Ga-FAPI-04 activity was distributed around the IUD ( $g$ and $h$, SUVmax 10.1). The red arrows point to the uterus. 

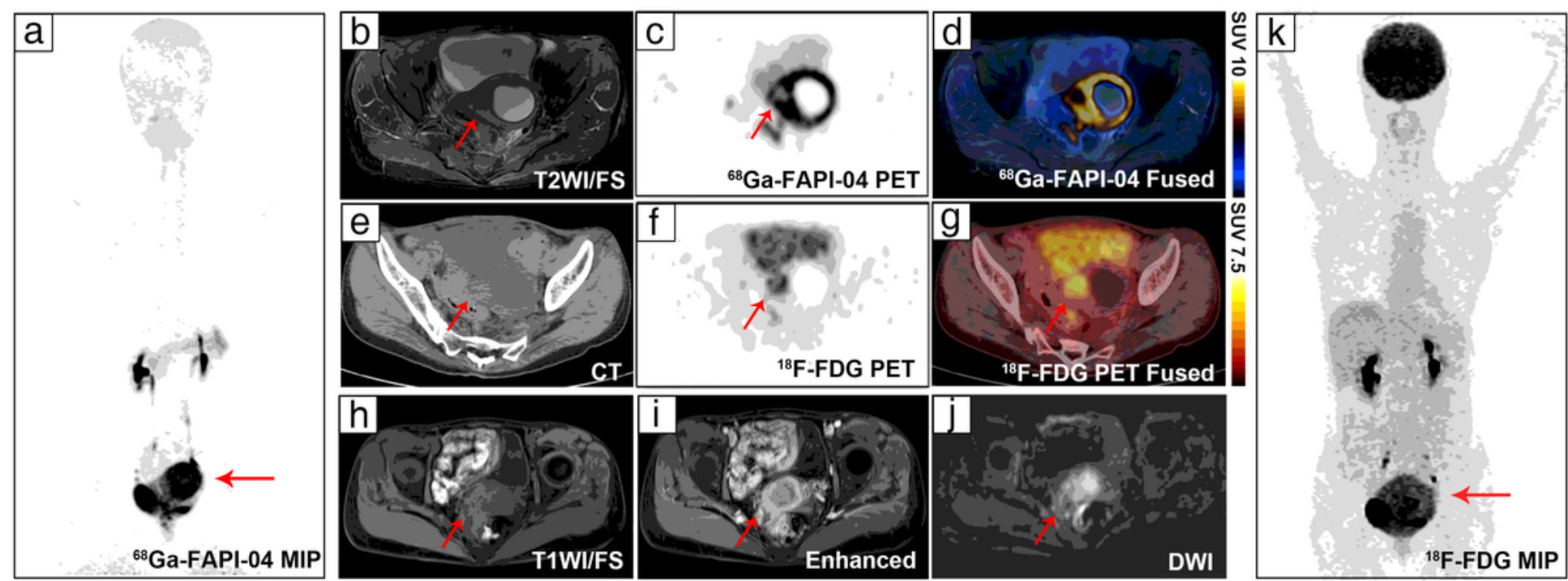

\section{Figure 4}

A woman of reproductive age had undergone gastrectomy with poorly differentiated gastric adenocarcinoma 4 years prior to scanning. Due to increasing abdominal pain, 68Ga-FAPI-04 PET/MR was performed for restaging. The MIP image (a) of a 68Ga-FAPI-04 PET/MRI showed the uterus (red arrow) had elevated activity with an SUVmax of 16.1. In tomographic T2WI/FS, PET, and fusion images (b-d, red arrows), a uterine mass $(4.3 \mathrm{~cm} \times 3.1 \mathrm{~cm} \times 3.7 \mathrm{~cm})$ was found with hypointense T2 signal and a relative lower 68Ga-FAPI-04 uptake (SUVmax 6.0, red arrows), while avid accumulation was shown in contemporaneous18F-FDG PET/CT images (SUVmax 8.1, e- $\mathrm{g}$ and $\mathrm{k}$, red arrows). The lesion became larger $(5.3 \mathrm{~cm} \times 4.0 \mathrm{~cm} \times 4.7 \mathrm{~cm})$ in the follow-up MRI imaging with enhancement and restricted diffusion ( $h-j$, red arrows), which implied it to be a malignancy.
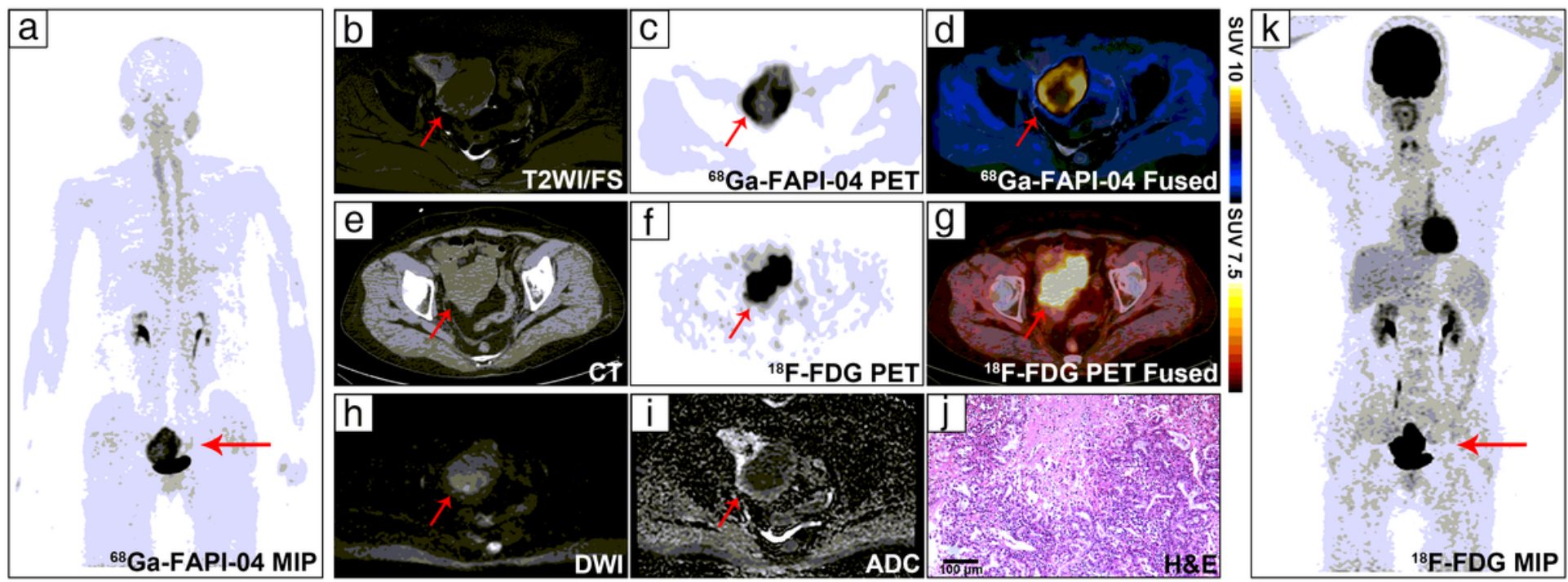

Figure 5

A postmenopausal female was examined for elevated tumor markers for 1 month. 68Ga-FAPI-04 and 18F-FDG PET both showed avid uptake in the uterus (a and k, SUVmax 13.4 and 21.7, respectively). The 
uterus was also noted to have slightly inhomogeneous T2 signal and restricted diffusion ( $b, h$, and i) with a size of $4.8 \mathrm{~cm} \times 6.6 \mathrm{~cm} \times 7.0 \mathrm{~cm}$. Hematoxylin-eosin (H\&E) staining manifested it as endometrial adenocarcinoma (j). The red arrows point to the uterus.
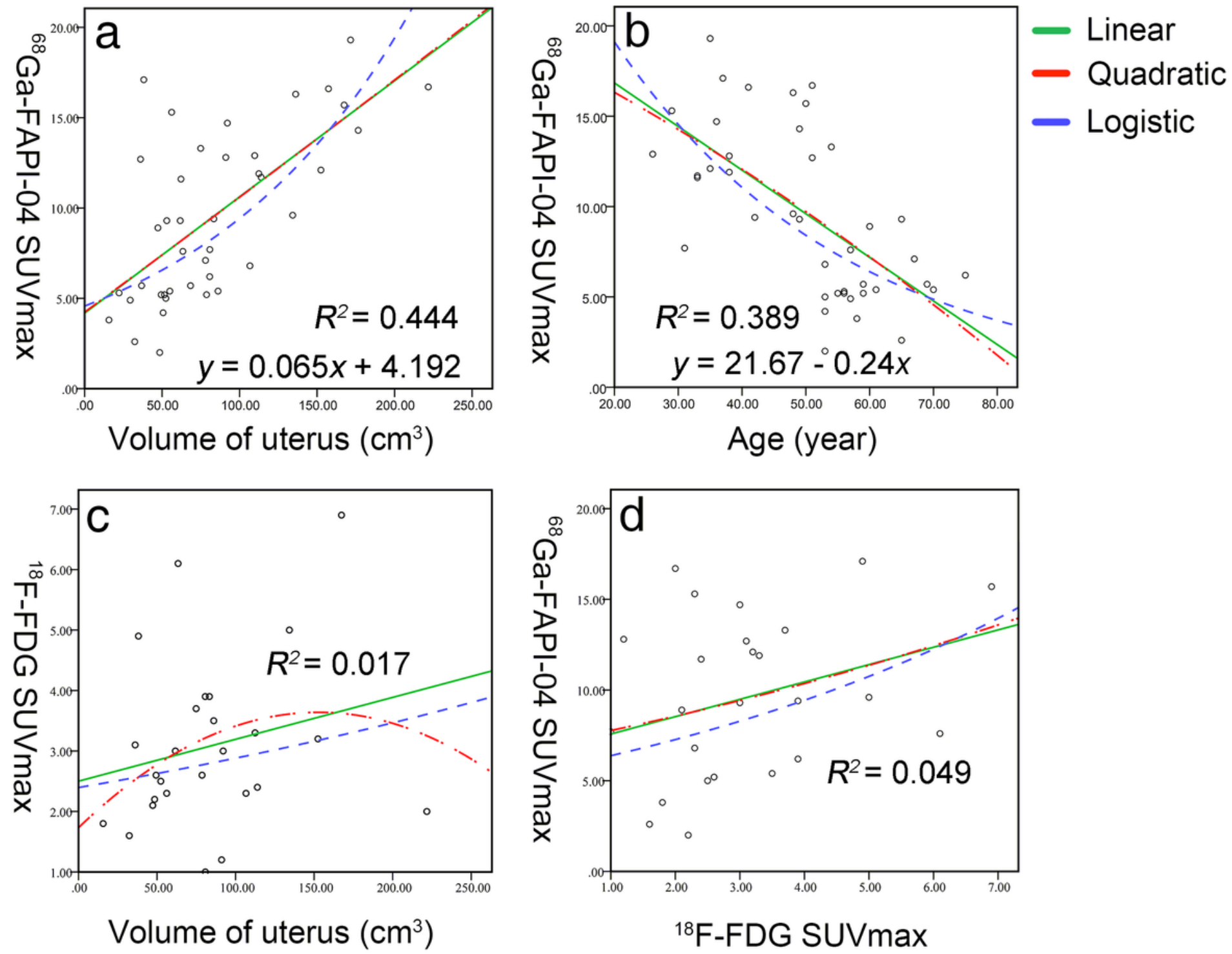

${ }^{18}$ F-FDG SUVmax

Figure 6

The relationships of continuous variables analyzed with curves fit by linear, quadratic, and logistic regression. The uterine size plotted against 68Ga-FAPI-04 uptake (a). Age plotted against 68Ga-FAPI-04 uptake (b). Uterine size (c) and 68Ga-FAPI-04 uptake (d) plotted against 18F-FDG uptake, respectively.

\section{Supplementary Files}

This is a list of supplementary files associated with this preprint. Click to download.

- Graphicalabstract.docx 